\title{
Time-Domain Ab Initio Study of Charge Relaxation and Recombination in Dye-Sensitized $\mathrm{TiO}_{2}$
}

\author{
Walter R. Duncan, Colleen F. Craig, Oleg V. Prezhdo*
}

\author{
Department of Chemistry \\ University of Washington \\ Seattle, WA 98195-1700
}

${ }^{*}$ Corresponding author. E-mail: prezhdo@u.washington.edu

\section{Complete Reference}

92. Z. Zhang, P. Fenter, L. Cheng, N. C. Sturchio, M. J. Bedzyk, M. Predota, A. Bandura, J. D. Kubicki, S. N. Lvov, P. T. Cummings, A. A. Chialvo, M. K. Ridley, P. Benezeth, L. Anovitz, D. A. Palmer, M. L. Machesky, and D. J. Wesolowski, Langmuir, 2004, 20, 4954. 